\title{
The Place of Protease Inhibitors in Antiretroviral Treatment
}

\author{
Tenore, S.B. and Ferreira, P.R.A. \\ Infectology Subject of the Federal University of São Paulo; São Paulo, SP, Brazil
}

\begin{abstract}
With the introduction of highly active antiretroviral therapy, a number of drugs have been developed. The best choice concerning which antiretroviral analogs to start is always under discussion, especially in the choice between non-nucleoside reverse transcriptase inhibitors-based therapies and ritonavir-boosted protease inhibitors. Both are proven to control viral replication and lead to immunological gain. The choice between a non-nucleoside analog reverse transcriptase inhibitor and a protease inhibitor as a third antiretroviral drug in the therapy should consider factors related to the individual, as well as the inclusion of the best therapy in the patient's daily activities and potential adherence. The protease inhibitor-based therapies showed similar efficacy among the various inhibitors with characteristics concerning the adverse events from each medicine. For the treatment of protease-resistant patients, darunavir and tipranavir showed good efficacy with higher genetic barrier to resistance.

Key-Words: AIDS, antiretroviral treatment, protease inhibitors.
\end{abstract}

Since 1996, with the introduction of highly active antiretroviral (ARV) therapy (HAART), a number of drugs have been developed. The best choice concerning which antiretroviral drugs to start is always under discussion, especially in the choice between non-nucleoside analog reverse transcriptase inhibitors (NNRTI)-based therapies and ritonavir-boosted protease inhibitors (PI/r). Both are proven to control viral replication and lead to immunological gain [1].

Different recommendations, based on national [2] and international [3-5] guidelines, indicate first choice and alternative drugs, in case of impossibility to use the preferable ones.

The choice between an NNRTI or a PI/r as the third antiretroviral drug in the therapy should consider factors related to the individual, such as: neuropsychiatric diseases, family planning (in case o female patients), cardiovascular risk (dyslipidemia and resistance to insulin), liver diseases, drugdrug interaction, as well as the inclusion of the best therapy in the patient's daily activity and potential adherence.

The Brazilian consensus for the treatment of HIV-infected adults and adolescents [2], recommends two NRTIs (zidovudine and lamivudine) in association with efavirenz or lopinavir/r. Among the alternative therapies associated with NRTI, it is possible to choose nevirapine or atazanavir/r (Table 1 ).

Table 1. Preferable and alternative recommendations from the Brazilian Consensus for ARV treatment.

\begin{tabular}{lll}
\hline & First Choice & Alternative \\
\hline 2 NRTI & AZT/3TC & ddI EC/3TC \\
& & TDF/3TC \\
NNRTI & Efavirenz & Nevirapine \\
PI & Lopinavir/r & Atazanavir/r \\
\hline ddI EC: didanisone "enteric coat" (slow release tablets). \\
TDF: tenofovir.
\end{tabular}

Received on 15 June 2009; revised 31 July 2009.

Address for correspondence: Dr. Tenore. Rua Loefgren 1588, Vila Clementino, CEP 04040-002 São Paulo/SP.

The Brazilian Journal of Infectious Diseases

2009;13(5):371-374. (C) 2009 by The Brazilian Journal of Infectious Diseases and Contexto Publishing. All rights reserved.
Currently, lopinavir/r represents the comparator PI in most of the large studies with new $\mathrm{PI} / \mathrm{r}$, in determining efficacy. It is an ARV with proven long-term efficacy [6], the only PI coformulated with ritonavir, representing a great advantage in terms of adherence and guarantee of use for ritonavir but, on the other hand, it does not allow a dose reduction of the latest in case of intolerance or metabolic changes.

Among the international guidelines, as recommended by the US Department of Health-DHHS, when it is decided to use a PI/r, the choice can be done between lopinavir/ritonavir (LPV/ r) once or twice daily, atazanavir/ritonavir (ATV/r) once daily, fosamprenavir/ritonavir twice daily and, most recently incorporated, darunavir/ritonavir (DRV/r) once daily. The latest, based on the ARTEMIS [7] study results, demonstrated DRV/ r non-inferiority compared to LPV/r, in antiretroviral therapy naïve patients over 48 weeks, and to be superior to LPV/r in 96 weeks follow-up. On the other hand, the International AIDS Society (IAS) [4] recommendation also includes the use of saquinavir/ritonavir (SQV/r) as the preference PI/r (Table 2).

\section{Factors to Be Considered When Choosing a PI/r in Initial Therapy}

All the protease inhibitors, except nelfinavir, should be administered with ritonavir. The coadministration increases the PI serum level, extending the dose intervals, facilitating the dosage, decreasing the number of tablets and increasing the strength, inclusively against strains with decreased sensitivity to PI $[8,9]$. The clinical trials with $\mathrm{PI} / \mathrm{r}$ showed they are comparable concerning the virological, immune responses and development of resistance [7,10-13].

\section{CASTLE Study: Atazanavir/r versus Lopinavir/r [10]}

The CASTLE study showed the non-inferiority of ATV/r (300/100 mg, once daily) versus LPV/r (400/100 mg twice daily) combined with tenofovir/emtricitabine (TDF/FTC), in 883 naïve patients randomized at 1:1 to each group after 48 weeks. In the 96-week analysis ATV/r has shown to be superior to LPVr (Figure 1). However, ATV/r has shown better tolerability and lower incidence of discontinuation related to adverse events, what can have influenced the intention to treat analysis [14]. 
Table 2. Comparison between DHHS, IAS and EACS guidelines.

\begin{tabular}{llll}
\hline Antiretroviral Drug & DHHS $^{3}$ & IAS-USA $^{4}$ & EACS $^{\mathbf{4}}$ \\
\hline NRTI & Tenofovir/Emtricitabine & Abacavir/Lamivudine & Abacavir/Lamivudine \\
& & Tenofovir/Emtricitabine & Tenofovir/Emtricitabine \\
NNRTI & Efavirenz & Efavirenz & Efavirens/Nevirapine \\
PI & Atazanavir/ritonavir & Atazanavir/ritonavir & Atazanavir/ritonavir \\
& Darunavir/ritonavir & Darunavir/ritonavir & Fosamprenavir/ritonavir \\
& Fosamprenavir/ritonavir (twice daily) & Fosamprenavir/ritonavir & Lopinavir/ritonavir \\
& Lopinavir/ritonavir (once or twice daily) & Lopinavir/ritonavir & Saquinavir/ritonavir \\
& & Saquinavir/ritonavir & \\
\hline
\end{tabular}

Hyperbilirubinemia was most common in patients using ATV/ $r$, and events related to the gastrointestinal (GI) tract, hypertriglyceridemia, hypercholesterolemia (including increased LDL) and the use of hypolipemiant agents was higher in patients using LPV/r (Figure 2).

Atazanavir should be used with caution when combined with antiacids and $\mathrm{H} 2$ blockers. The advantages of using atazanavir are good gastrointestinal tolerance, the lower number of tablets and the once daily use, improving adherence. It should be preferably administered with food.

\section{KLEAN Study [11]: Fosamprenavir/r versus Lopinavir/r}

The association FPV/r (700/100 mg twice daily) showed to be non-inferior when compared with LPV/r (400/100 mg twice daily), in combination with abacavir/lamivudine (ABC/3TC) in 878 antiretroviral therapy naïve patients, in 144 weeks (Figure 3). The tolerability and incidence of adverse events were similar between the groups.

In a retrospective study, Calza et. al. showed that fosamprenavir/ritonavir (700/100 mg twice daily) has similar efficacy in virological and immune responses when compared to lopinavir/ritonavir at the usual dose, both associated with two NRTIs, but with lower incidence of diarrhea and hypertriglyceridemia when fosamprenavir/ritonavir was used as the third drug in ARV therapy ( $\mathrm{p}=0,006$ and $\mathrm{p}=0,008$, respectively) [15].

\section{ALERT Study [12]: Fosamprenavir/r versus Atazanavir/r}

In this study, 106 naïve patients were randomized to receive FPV/r (1400/100 mg once daily) or ATV/r (300/100 mg once daily) associated with TDF/FTC. The virological response in 48 weeks was similar between the groups. Adverse events were more common in patients using ATV/r, especially due to hyperbilirubinemia. Lipid changes were similar between the groups (Figures 4 and 5).

FPV/r has the advantage of being administered once or twice daily, without food restriction. In association with 100 mg RTV once daily, it seems to have a better metabolic profile, when compared with $200 \mathrm{mg} /$ day RTV [16].

The use of FPV/r (1400/100 mg once daily) was approved by FDA; however, it remains as an alternative recommendation by DHHS, probably, due to the existence of few studies validating this dose.

\section{GEMINI Study [13]: Saquinavir/ritonavir versus Lopinavir/ Ritonavir}

This study compared the use of SQV/r (1000/100 mg twice daily) and LPV/r (400/100 mg twice daily) in 337 antiretroviral therapy naïve patients, and showed the non-inferiority of SQV/ r compared to LPV/r (Figure 6). SQV/r was associated with a lower increase in triglycerides, total cholesterol (but not concerning LDL) and similar occurrence of other adverse events. However, the significant amount of tablets (10 tablet/day SQV) makes the use of this drug inconvenient compared to other PIs.

\section{ARTEMIS Study [6]: Darunavir/Ritonavir versus Lopinavir/ Ritonavir}

In this study, involving 689 individuals, DRV/r (800/100 mg once daily) showed to be non-inferior when compared to LPV/ r (400/100 mg once daily or 800/200 mg once daily), after 48 weeks of treatment, in ARV treatment naïve patients. DRV/r was superior in the subgroup with viral load (VL) higher than 100,000 copies/mL. The 96-week analysis also showed that DRV/r is superior for patients with VL lower than 50 copies/mL, being this difference caused, especially, by the lower virological failure in this group. DRV/r showed lower incidence of adverse events related to GI tract and lower lipid changes (Figures 7 and 8).

\section{Antiretroviral Treatment in Patients with Resistance to Multiple Drugs}

In patients multi-experienced with PIs, the shift to another ARV therapy should be oriented by genotype and should contain a ritonavir-boosted protease inhibitor, even with protease resistance [2]. The decreased PI activity, as well as with NRTIs, is not complete, even in the presence of resistance mutations, due to the residual effect that these drugs still maintain [17,18].

Darunavir and tipranavir are non-peptide protease inhibitors, with increased affinity to the active protease site, with bindings through stronger hydrogen bridges $[19,20]$. In vitro studies evidenced that the resistance to darunavir was developed on a slower way compared with other PIs.

The POWER studies [21] showed the efficacy of using darunavir/ $\mathrm{r}$ in patients with antiretroviral experience in three classes and documented resistance in protease. In this study, 255 patients were randomized at a 1:1 ratio to receive darunavir/ r (600/100 mg twice daily) or a PI/r as a comparator, chosen by 
Figure 1. Virological response, CASTLE study: atazanavir/ ritonavir versus lopinavir/ritonavir in 48 and 96 weeks (ITT analysis).

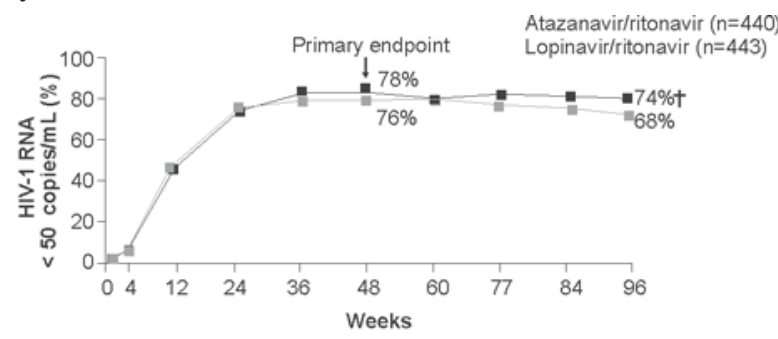

*Atazanavir/rtonavir noninferior to lopinavir/ritonavir tAtazanavir/rtonavir superior to lopinavir. $\mathrm{P}<05$

Permission received by Clinical Care Options for use of the this figure.

Figure 2. CASTLE: lipid changes in week 96.

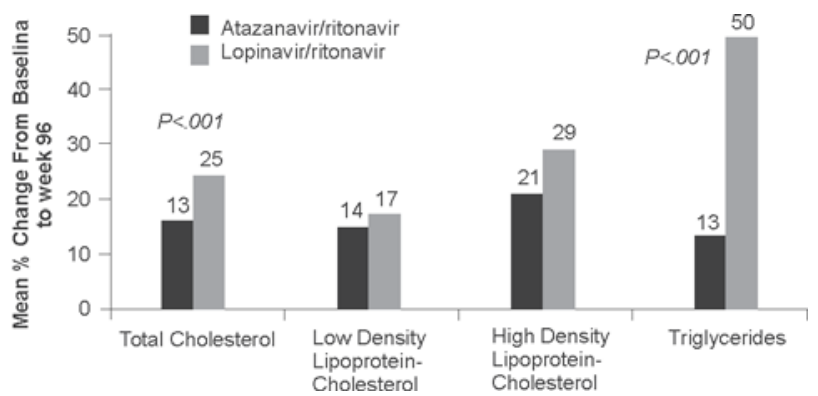

Figure 3. Virological response in study KLEAN: fosamprenavir/ ritonavir versus lopinavir/ritonavir-144 weeks (ITT analysis).

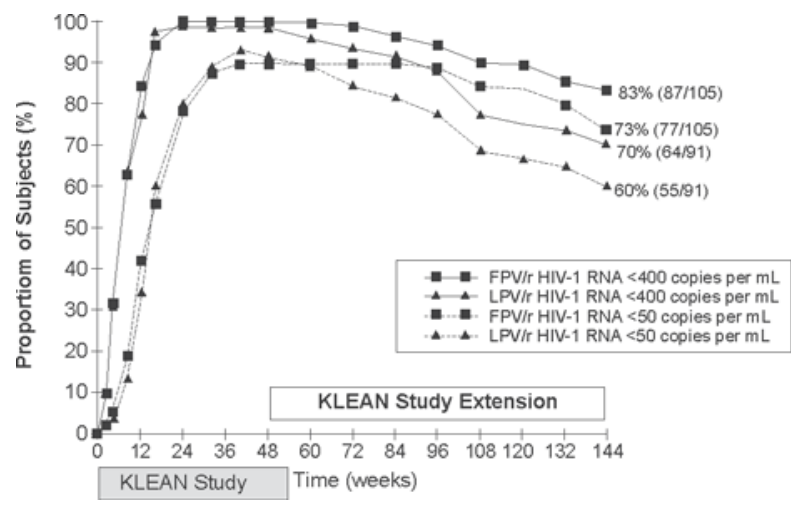

Figure 4. Virological response in study ALERT: fosamprenavir/ ritonavir versus atazanavir/ritonavir in 48 weeks (ITT analysis).

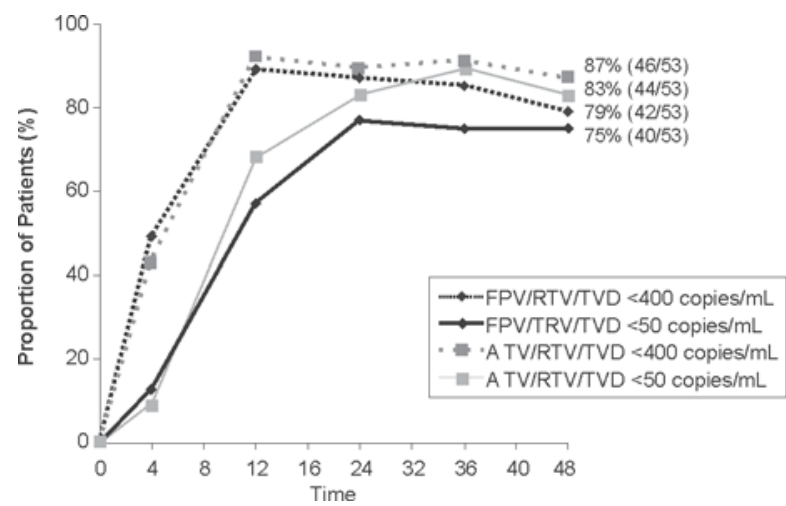

Figure 5. ALERT: lipid changes in 48 weeks.

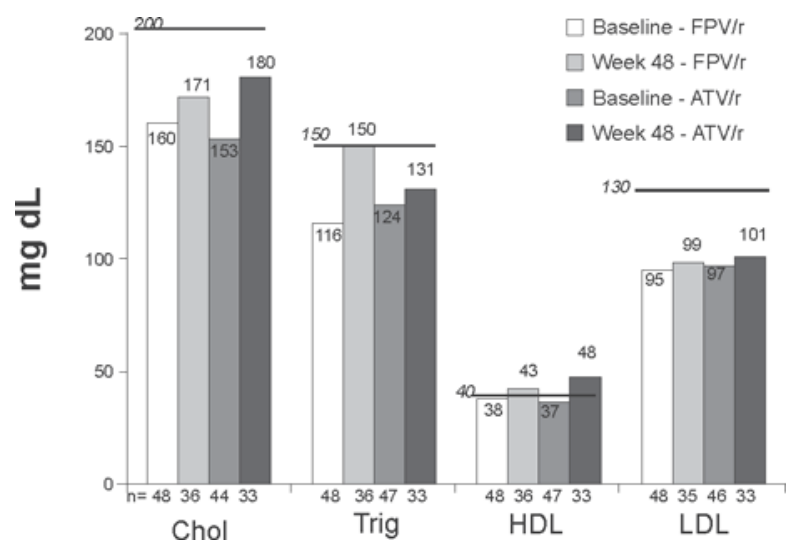

Figure 6. Virological response, study GEMINI: lopinavir/ ritonavir versus saquinavir/ritonavir in 48 weeks.

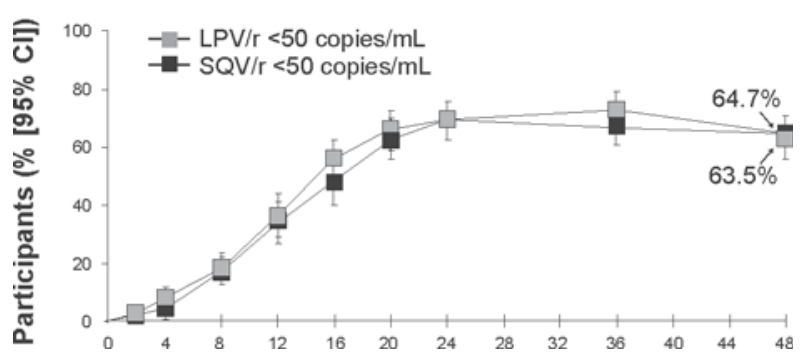

Figure 7. Virological response in study ARTEMIS: darunavir/ ritonavir versus lopinavir/ritonavir in 48 and 96 weeks.

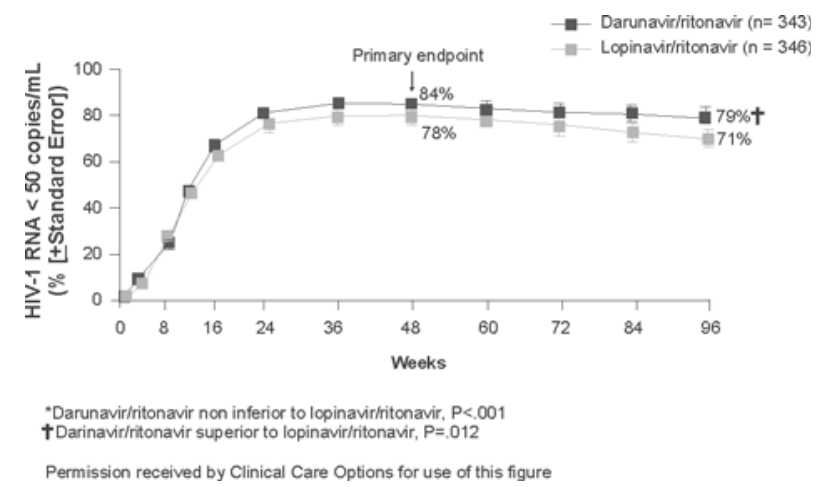

Figure 8. ARTEMIS: Lipidic changes in 96 weeks.

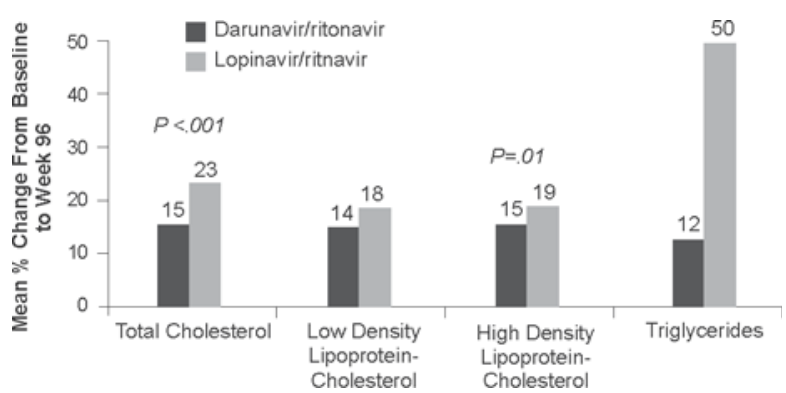


Figure 9. Virological response in study POWER in 144 weeks.

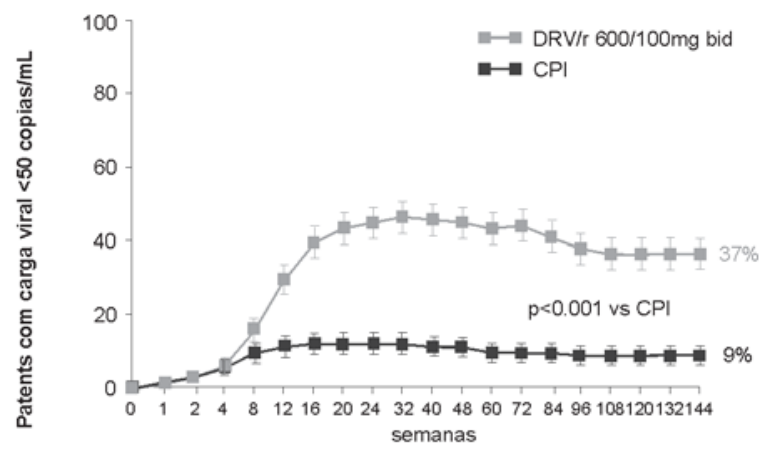

Katama C. ot al. ght ICDTH1 2008. Abstract P21

the investigator after being oriented by resistance tests, associated with an optimized base therapy. As shown in Figure 9, in 144 weeks $37 \%$ of the individuals using DRV/r had a viral load that was lower than 50 copies $/ \mathrm{mL}$, compared to $9 \%$ of the patients using another $\mathrm{PI} / \mathrm{r}$. In the same analysis, the gain with CD4 was also superior in the group treated with DRV/r.

The use of tipranavir in multi-experienced patients was shown in RESIST studies, which included 1483 patients with previous use of the three ARV classes, also with documented resistance in protease. The participants were randomized to receive tipranavir/r or one of the 4 comparator $\mathrm{PI} / \mathrm{r}$ : LPV/r. $\mathrm{SQV} / \mathrm{r}$, indinavir/r or amprenavir/r. After 48 weeks of treatment, $33.6 \%$ of the patients receiving tipranavir/ $\mathrm{r}$ and $15.3 \%$ of those receiving the $\mathrm{PI} / \mathrm{r}$ comparator had a decrease in $\mathrm{VL}$ that was higher than $1 \log 10$ (primary study endpoint). Concerning a VL lower than 400 copies $/ \mathrm{mL}, 30.4 \%$ versus $13.8 \%$ of the patients reached this endpoint in tipranavir/r and $\mathrm{PI} / \mathrm{r}$ comparator groups, respectively $(\mathrm{p}<0,0001)$ [22].

In highly active antiretroviral treatment, the ritonavirboosted protease inhibitors have a key role, both in naïve patients, as well in those with antiretroviral failure, due to their high genetic barrier and proven long term efficacy. As a initial therapy, despite of the adverse events related to the metabolic syndrome, it allows an efficient treatment, with low potential to develop resistance. The virological and immune responses are similar among the various available $\mathrm{PI} / \mathrm{r}$, as shown in randomized clinical studies. The various PI/r options have their particularities concerning toxicity and dosage administration, which should be considered when choosing a PI to use, in accordance with each clinical case. In antiretroviral rescue, even with the advent of new classes, such as raltegravir, etravirine and maraviroque, $\mathrm{PI} / \mathrm{r}$ should still be used in therapy, according with resistant tests, once they present a residual activity event in the presence of mutation, besides the fact that all rescue clinical studies highlight the importance of using this class of drugs.

\section{References}

1. Cuzin L., Allavena C., Morlat P., Dellamonica P. Boosted protease inhibitors-based or nonnucleoside reverse transcriptase-based HAART: is there a best choice for antiretroviral -naïve HIV-1 infected patients? AIDS Rev 2008;10(4):205-11.
2. Recomendações de terapia antiretroviral para adultos e adolescentes infectados pelo HIV 2008-2009. www.aids.gov.br em documentos e publicações. [acessado em abril 2009].

3. Guidelines for the use of antiretrovirals agents in HIV-1 adults and adolescents. Desenvolvido pelo DHHS panel on antiretroviral guidelines for adults and adolescents. Nov, 2008. http:// aidsinfo.nih.gov/ContentFiles/AdultandAdolescentGL.pdf.

4. Hammer S. et al. IAS-USA guideline. JAMA 2008;300:555-70.

5. European guidelines for treatment of HIV infected adults in Europe. http://www.eacs.eu/guide.

6. Barragan P., Podzamczer D. Lopinavir/ritonavir: a protease inhibitor for HIV treatment. Expert Opin Pharmacother. 2008;Sep;9(13):2363-75.

7. Mills A., Nelson M., Jayaweera D. et al. Efficacy and safety of darunavir/ritonavir 800/100 mg once-daily versus lopinavir/ ritonavir in treatment-naive, HIV-1-infected patients at 96 weeks: ARTEMIS (TMC114-C211). Program and abstracts of the 48th Annual ICAAC/IDSA 46th Annual Meeting; October 25-28, 2008; Washington, DC. Abstract H-1250c.

8. Youle M. Overview of boosted protease inhibitors in treatment experienced HIV-infected patients. J Antimicrob Chemother 2007 Dec;60(6):1195-205.

9. Wilkins E.J. The current role of ritonavir-boosted protease inhibitors in the management of HIV infection. HIV Ther 2008 Mar;13(1):9-18. Review.

10. Molina J.M., Andrare-Villanueva J., Echevarria J. et al. Atazanavir/ ritonavir vs. lopinavir/ritonavir in antiretroviral-naive HIV-1-infected patients: CASTLE 96 week efficacy and safety. Program and abstracts of the 48th Annual ICAAC/IDSA 46th Annual Meeting; October 25-28, 2008; Washington, DC. Abstract H-1250d.

11. Pulido F. et. al. Long term efficacy and safety of fosamprenavir plus ritonavir versus lopinavir/ritonavir in combination with abacavir/ lamivudine over 144 weeks. HIV Clin. Trials 2009;10(2):76-87.

12. Smith K.Y., Weinberg W.G., Dejesus E. et al. Fosamprenavir or atazanavir once daily boosted with ritonavir $100 \mathrm{mg}$, plus tenofovir/emtricitabine, for the initial treatment of HIV infection: 48-week results of ALERT. AIDS Res Ther 2008;5:5.

13. Walmsley S.L. et al. Gemini: a noninferiority study of saquinavir/ ritonavir versus lopinavir/ritonavir as initial HIV-1 therapy in adults. JAIDS 2009;50(4):367-74.

14. Molina J.M., Andrare-Villanueva J., Echevarria J. et al. Atazanavir/ ritonavir vs. lopinavir/ritonavir in antiretroviral-naive HIV-1-infected patients: CASTLE 96 week efficacy and safety. Program and abstracts of the 48th Annual ICAAC/IDSA 46th Annual Meeting; October 25-28, 2008; Washington, DC. Abstract H-1250d.

15. Calza L. et al. Efficacy and tolerability of a fosamprenavir/ritonavirbased versus lopinavir/ritonavir-based antiretroviral treatment in 82 therapy-naïve patients with HIV-1 infection. International Journal of STD\&AIDS 2008;19:541-4.

16. DeJesus E., Sloan L., Sension M. et al. 96-week efficacy/safety data comparing two doses of ritonavir (/r) to boost once-daily (QD) fosamprenavir (FPV), used in combination with abacavir (ABC)/ lamivudine (3TC). Program and abstracts of the 48th Annual ICAAC/IDSA 46th Annual Meeting; October 25-28, 2008; Washington, DC. Abstract H-1246.

17. Castagna A. et al. Lamivudine monotherapy in HIV-1-infected patients harbouring a lamivudine-resistant virus: a randomized pilot study (E-184V study). AIDS 2006;20(6):795-803.

18. Deeks S.G. et al. Interruption of treatment with individual therapeutic drug classes in adults with multidrug-resistance HIV1 infection. J. Infec Dis 2005;192(9):1537-44.

19. Lefebvre E., Schiffer C.A. Resilience to resistance of HIV-1 protease inhibitors: profile of darunavir. AIDS Rev 2008;10(3):131-42.

20. Walmsley S.L. et al. Pharmacokinetics, Safety, and Efficacy of Tipranavir Boosted With Ritonavir Alone or in Combination With Other Boosted Protease Inhibitors as Part of Optimized Combination Antiretroviral Therapy in Highly Treatment-Experienced Patients (BI Study 1182.51). JAIDS 2008;47(4):429-40.

21. Katlama C. et al. Power I and II: combined final 144-week efficacy and safety results for Prezista/r 600/100 mg bid in treatmentexperienced HIV patients 9th ICDTHI 2008. Abstract P21

22. Walmsley S.L. et al. Multidrug-experienced HIV-1-infected women demonstrated similar virological and immunological responses to tipranavir/ritonavir compared with men. AIDS 2009;23(3):429-31. 